\title{
Microstructure Identification Based on Vessel Pores Feature Extraction of High-value Hardwood Species
}

\author{
Xiaoxia Yang, Ziyu Zhao, Zhongmin Wang, Zhedong Ge,** and Yucheng Zhou
}

\begin{abstract}
Because of the diversity of vessel pores in different hardwood species, they are important for wood species identification. In this paper, a Micro CT was used to collect wood images. The experiment was based on six wood types, Pterocarpus macrocarpus, Pterocarpus erinaceus, Dalbergia latifolia, Dalbergia frutescens var. tomentosa, Pterocarpus indicus, and Pterocarpus soyauxii. One-thousand cross-sectional images of $2042 \mathrm{px} \times$ $1640 \mathrm{px}$ were collected for each species. One pixel represents $1.95 \mu \mathrm{m}$ of the real physical dimension. The level set geometric active contour model was used to obtain the contour of the vessel pores. Combined with a variety of morphological processing methods, the binary images of the vessel pores were obtained. The features of the binary images were extracted for classification. Classifiers such as BP neural network and support vector machine were used, the number, roundness, area, perimeter, and other characteristic parameters of the vessel pores were classified, and the accuracy rate was more than $98.9 \%$. The distribution and arrangement of the vessel pores of six kinds of hardwood were obtained through the level set geometric active contour model and image morphology. Then BP neural network and support vector machine were used for realizing the classification of hardwood species.
\end{abstract}

Keywords: Vessel pores; Level set; BP neural network; RBF Kernel SVM classifier

Contact information: School of Information and Electrical Engineering, Shandong Jianzhu University, Jinan, Shandong 250101, China; *Corresponding author: gezhedong@163.com

\section{INTRODUCTION}

Study of wood classification and recognition allows better understanding of its biological structural characteristics and physical properties for proper use (Wang et al. 2013; Beeckman 2016). Traditionally, the identification and appraisal of wood microstructure are performed by experienced experts by identification of the wood internal structure (Kamal et al. 2017; Rajagopal et al. 2019). As machine vision and pattern recognition technology have developed, wood identification methods have changed from manual identification to intelligent means, such as machine learning. For example, both Souza et al. (2020) and Zamri et al. (2016) extracted texture features to classify wood with different classifiers, and good classification results were achieved. In addition, $\mathrm{Hu}$ et al. (2015) and Rajagopal et al. (2019) used a SIFT (scale invariant feature transform) algorithm to extract key points from wood cross-section images, and they used image deblurring and feature extraction methods to classify and recognize wood via a support vector machine.

Though several scholars have performed wood recognition and classification according to wood texture, color, and other features, there are other methods to identify wood such as a structure of wood, such as vessel pores, ray, through segmentation, image processing, morphological analysis, and other methods. The vessel pores only exist in 
hardwood, and it is the main structure that distinguishes coniferous wood from hardwood (Ruffinatto et al. 2015). Chen and Evans (2010) used SilviScan technology to complete near infrared spectrum radial scanning of birch and poplar combined with a series of image processing and statistical analysis technology to identify and measure the birch and poplar vessel pores. Mallik et al. (2011) collected images of seven types of wood and magnified them 1500 times by SEM (scanning electron microscopy). After segmentation, tracheids feature extraction, and classification by different classifiers, the results showed that KNN (K-nearest neighbor) and neural network were the best classification methods, and their accuracy rate was over $93 \%$. The tracheids, pores, and wood rays of several Canadian coniferous and broadleaf woods were segmented with a watershed algorithm by Brunel et al. (2014), and the results showed that the watershed algorithm completed cell segmentation and recognition faster than manual segmentation. Images of 30 kinds of wood were magnified 10 times and processed with homomorphic filtering technology. Then, the images were binary processed with the Otsu optimal threshold, and the vessel pores features were extracted. After neural network classification, the accuracy rate reached 89.3\% (Ibrahim et al. 2018). Zhao and Wang (2019) extracted the spectral features with a mathematical morphology operation and K-L divergence at the edge and center region of the wood vessel pores. After recognition with three dimensionality reduction algorithms and three classifiers, the results showed that PCA (principal component analysis) and principal component analysis MDS (multidimensional scaling) combined with an MD (Mahalanobis distance) algorithm gave the best recognition answers.

Most of the cited scholars extracted tracheids and vessel pores through segmentation. In this study, the level set geometric active contour method was used to obtain the contour of the vessel pores (Li et al. 2010; Xin et al. 2012). This method is extensively used in medical image segmentation field, and it has yet to be applied in the wood image segmentation field. In this study, this method was used to determine the contour of the wood vessel pores. First, the contour of the vessel pores was determined with the level set method. Then, binary images of the vessel pores were combined with image morphology processing, and the vessel pores characteristic parameters were extracted. Then, classification and recognition for six kinds of broad-leaved woods were performed with a BP neural network and the radial basis function (RBF) Kernel support vector machine (SVM).

\section{EXPERIMENTAL}

\section{Materials}

The experimental materials were selected from the Specimens Museum of the Shandong University of Architecture (Jinan China). Six species of high-value Papilionaceae hardwood were selected, and the sizes of the prepared specimens were $2 \mathrm{~mm}$ $\times 2 \mathrm{~mm} \times 15 \mathrm{~mm}$. A Bruker kyScan1272 micro CT scanner was employed (Bruker Corporation, Antwerp, Belgium). The voltage and current of the ray source were set to 50 $\mathrm{kV}$ and $200 \mathrm{uA}$, respectively. The projection data was acquired with an angle of $0.1^{\circ}$. One thousand images of $2042 \mathrm{px} \times 1640 \mathrm{px}$ in size were reconstructed for each specimen. The image resolution of each kind of wood is $1.95 \mu \mathrm{m}$, which means that a pixel represents $1.95 \mu \mathrm{m}$ actual physical dimension. For each specimen, 100 images were randomly selected from the reconstructed mages, which were cut to $700 \mathrm{px} \times 700 \mathrm{px}$ as the training samples. The specific experimental material information is shown in Table 1. 
Table 1. Designation of the Experimental Materials and Characteristics of the Vessel pores

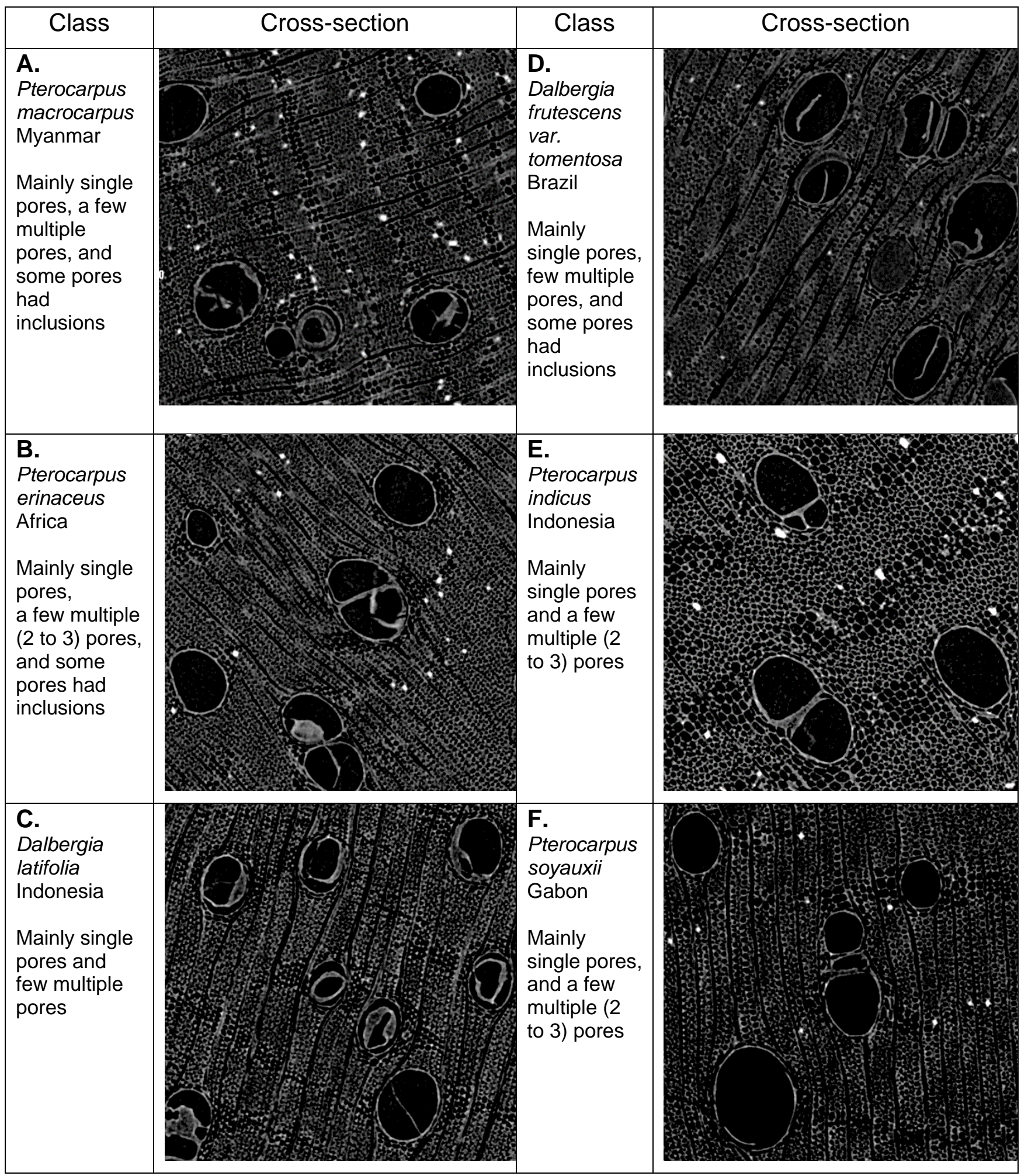




\section{Methods}

Vessel pores feature extraction

In this study, the contour of the vessel pores was determined by the level set active contour model (Li et al. 2010; Xin et al. 2012). According to the evolution curve, the contour of the vessel pores was calculated with $C(s, t)=(x(s, t), y(s, t))$, where $s$ is the parameter variable of the curve, $t$ is the time variable, and the curve of the geometric active contour model $(C)$ was expressed as (1),

$$
\frac{\partial C}{\partial t}=g\left(V_{o}+K\right) N
$$

where $N$ is the inward unit normal vector of the contour line $(C), K$ is the curvature of the contour $(C), V_{0}$ is a constant coefficient, $g$ is a function dependent on the image features. As the definition of gray image was $I(x, y)$, the function $(x, y)$ was expressed as (2),

$$
g(x, y)=\frac{1}{1+\left|\nabla\left(G_{\sigma} * I(x, y)\right)\right|^{2}}
$$

where $G_{\sigma}$ represents Gaussian kernal with standard deviation $\sigma$, * represents convolution, $I(x, y)$ represents the image to be segmented, and $\nabla$ is a spatial gradient operator. The active contour of the vessel pores in Fig. 1 was calculated with (2).

The gray image of vessel pores contour obtained above was transformed into binary image by threshold segmentation. Then the morphological operation was carried out to extract the vessel pores feature parameters. The specific process is shown below. First, the threshold was calculated automatically via the Otsu method (Ferman et al. 2002; Zhang et al. 2016), and the vessel pores and the background of the image were carved up. Then, the images erosion operation was successively with $3 \times 3$ structural elements and $5 \times 5$ structural elements in turn. Irrelevant information was removed when removing small targets. Finally, the $5 \times 5$ structural elements were used for the dilation operation twice, so that the vessel pores could be restored to the original size.

Based on the binary images of the six kinds of hardwood vessel pores, the vessel pores of seven characteristics (the vessel pores number $N$, the total vessel pores area $S_{\text {sum, }}$ the average vessel pores area $S_{\text {ave, }}$, the total vessel pores perimeter $P_{\text {sum }}$, the average vessel pores perimeter $P_{\text {ave, }}$, the total vessel pores roundness $C_{\text {sum, }}$ and the average vessel pores roundness $C_{\text {ave }}$ ) were counted. In each image, the vessel pores numbers were expressed as $x, x \in[1, N]$, where $N$ is the total number of vessel pores in each image, which was calculated by counting the number of connected domains in the image.

According to (3)-(5), each vessel pores area $\left(S_{\mathrm{x}}\right)$, the total vessel pores area $\left(S_{\text {sum }}\right)$, and the average vessel pores area $\left(S_{\text {ave }}\right)$ were calculated from the binary images.

$$
\begin{aligned}
& S_{x}=\sum_{i=1}^{N} a_{i 0}\left(y_{i-1}+\frac{1}{2} a_{i 2}\right) \\
& S_{\text {sum }}=\sum_{x=1}^{N} S_{x}=S_{1}+\cdots+S_{N} \\
& S_{\text {ave }}=\frac{1}{N} \sum_{x=1}^{N} S_{x}
\end{aligned}
$$

where $y_{\mathrm{i}}=y_{\mathrm{i}-1}+a_{i 2}, y_{0}$ is the ordinate of the initial point in (3), $a_{\mathrm{i} 0}$ and $a_{\mathrm{i} 2}$ are the length of the $i$ th ring of chain code in the direction of $k=0$ (horizontal) and $k=2$ (vertical), respectively. 
According to (6)-(8), the perimeter of each vessel pores could be calculated in a binary image and is expressed as $P_{\mathrm{x}}$, the total perimeter is expressed as $P_{\text {sum, }}$ and the average perimeter is expressed as $P_{\text {ave }}$,

$$
\begin{aligned}
& P_{x}=\sum_{i=1}^{N} \Delta l_{i}=n_{e}+\left(n-n_{e}\right) \sqrt{2} \\
& P_{\text {sum }}=\sum_{x=1}^{N} P_{x}=P_{1}+\cdots P_{N} \\
& P_{\text {sum }}=\sum_{x=1}^{N} P_{x}=P_{1}+\cdots P_{N}
\end{aligned}
$$

where the boundary chain codes of the vessel pores are set to $a_{1}, a_{2}, \ldots, a_{\mathrm{n}}$, the length of line corresponding to $a_{\mathrm{i}}$ is $\Delta l_{i}$, and the perimeter of the region's boundary was calculated using (6). In (6), $n_{e}$ is the number of even codes in the chain code sequence, and $n$ is the total number of codes in the chain code sequence.

According to (9)-(11), the roundness of each vessel pores is calculated in binary image and expressed as $C_{\mathrm{x}}$, and the total roundness and the average roundness are expressed as $C_{\text {sum }}$ and $C_{\text {ave }}$, respectively,

$$
\begin{aligned}
& C_{x}=\frac{4 \pi S_{x}}{P_{x}^{2}} \\
& C_{\text {sum }}=\sum_{x=1}^{N} C_{x}=C_{1}+\cdots+C_{N} \\
& C_{\text {ave }}=\frac{1}{N} \sum_{x=1}^{N} C_{x}
\end{aligned}
$$

where $S_{\mathrm{x}}$ is the area of the $x$-th vessel pores, $P_{\mathrm{x}}$ is the perimeter of the $x$-th vessel pores, If $C_{\mathrm{x}}$ is close to 1 , then the object is close to the circle. If $C_{\mathrm{x}}=1$, the detection object is a standard circle.

\section{Vessel pores classification}

BP neural network and support vector machine are very classic classification algorithms, and support vector machine is more used for small sample classification (Ruan et al. 2020; Mu et al. 2016). In this paper, basing on the seven characteristic parameters of the vessel, the training set data was $70 \%$, and the test set data was $30 \%$. The purpose was to realize the classification of hardwood. Parameter calculation and hardwood classification were completed by MATLAB 2016b. (Math Works, v.2016b version, Natick, MA, USA).

The network was trained with 7 input neurons, 6 output neurons, and 13 hidden layer neurons (Ding et al. 2014; Wang et al. 2019). The input layer and the hidden layer followed the Tansig tangent function. The hidden layer was the Purelin linear function of the output layer. The training number was 1000 , the learning efficiency was 0.01 , the error of training target was 0.00001 , and the momentum factor was 0.01 . The experimental results are shown in Table 3.

In this study, the LIBSVM software package (Taiwan, China) was used for classification and recognition, which is a classic classification toolbox for support vector machine (Chih and Lin 2011). There are four kinds of kernel functions, which include linear functions, polynomial functions, radial basis functions (RBF), and sigmoid kernel functions. In this study, the RBF kernel function was chosen to map a sample to a higher dimensional space so that the linear inseparable data became linearly separable. The 5-fold 
cross validation was employed to search for the best parameters of $c$ (penalty factor) and $g$ (variance in RBF kernel function). The experimental results are shown in Table 3.

\section{RESULTS AND DISCUSSION}

The images of the six kinds of hardwood cross-sections shown in Table 1 were collected by the Skyscan1272 micro CT scanner (Bruker Corporation, Antwerp, Belgium). The level set geometric contour model was used to process the images. The processed image differed substantially from the surrounding tissue structure, as shown in Fig. 1.

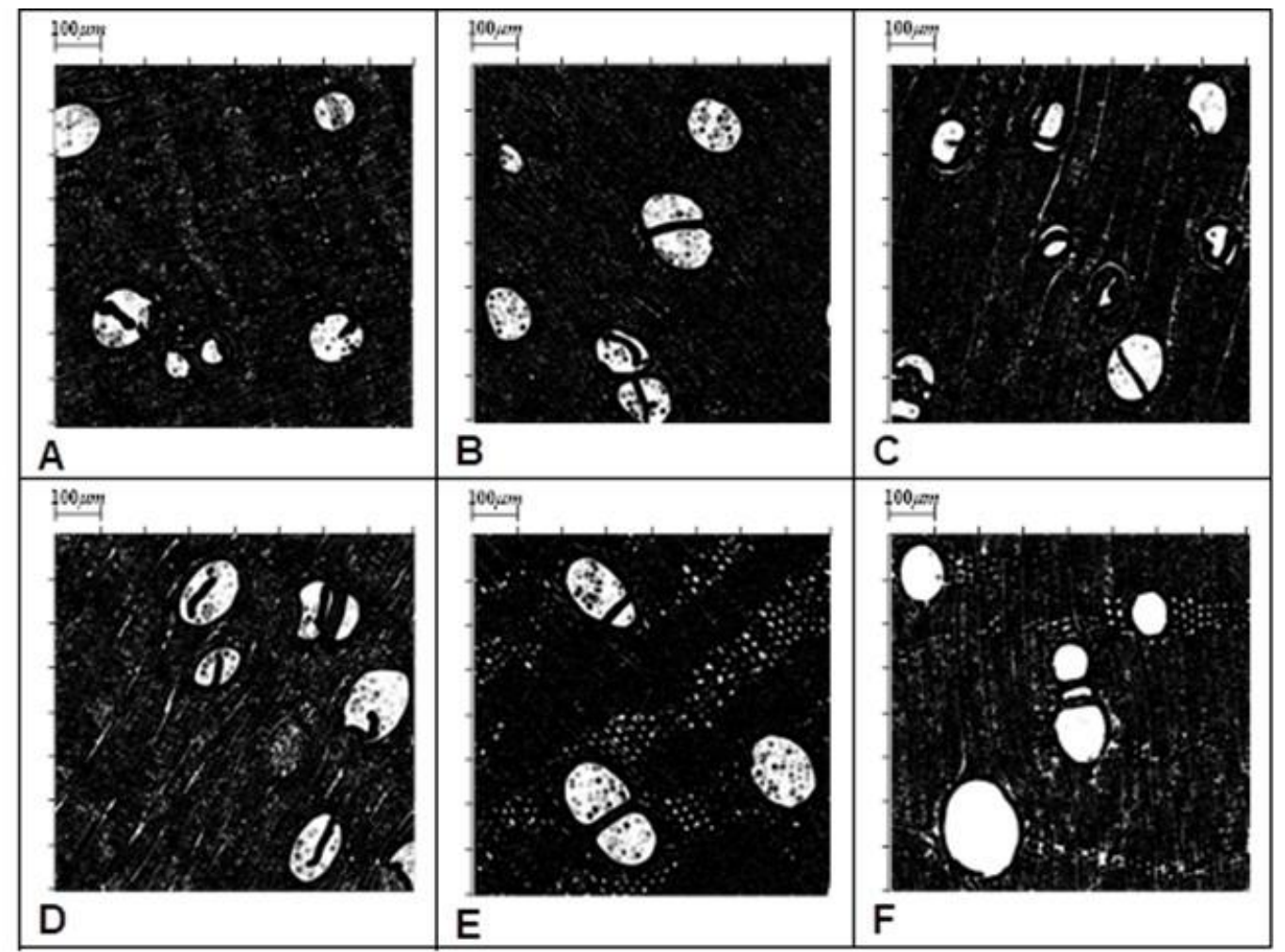

Fig. 1. The image processed by the level set geometric active contour model; A is Pterocarpus macrocarpus; B is Pterocarpus erinaceus; C is Dalbergia latifolia; D is Dalbergia frutescens; E is Pterocarpus indicus; F is Pterocarpus soyauxii

In Fig. 1A (Pterocarpus macrocarpus), the vessel pores were clear and contained some sediment. Due to the similarity of the gray value among the axial parenchyma cells, ray cells, and the vessel pores, the axial parenchyma cells and fine linear ray cells were still visible. In Fig. 1B (Pterocarpus erinaceus), the vessel pores were visible and contained more sediment. Because the gray values of the ray cells were similar to the vessel pores, the wood rays and several crystal cells within them were observed. In Fig. 1C (Dalbergia latifolia), the contours of the vessel pores were clear. Because the gray value of the contents was similar to the surrounding tissues, it was difficult to segment the vessel pores completely, and ray cells can be seen in the image. In Fig. 1D (Dalbergia frutescens), most of the vessel pores were clearly displayed and contained sediment. However, few vessel pores had many inclusions, so the vessel pores contour segmentation were unobvious, and 
part of the rays, axial parenchyma, and crystal cells were still visible. In Fig. 1E (Pterocarpus indicus), the vessel pores contours were clear, but there were a lot of sediments in the vessel pores. In addition, the axial parenchyma had a banded distribution, which is a characteristic structure of this variety. In Fig. 1F (Pterocarpus soyauxii), there were no inclusion in the vessel pores, so the contour was segmented well, and part of the axial parenchyma cells and ray cells were clear in the image.

Due to the vessel pores contour segmentation results in Fig. 1, erosion, small target removal, and dilation were used in sequence, according to the morphological processing method. Unrelated structures such as axial parenchyma cells, ray cells, and crystal cell structures were removed, and the binary image of the vessel pores were obtained, as shown in Fig. 2.

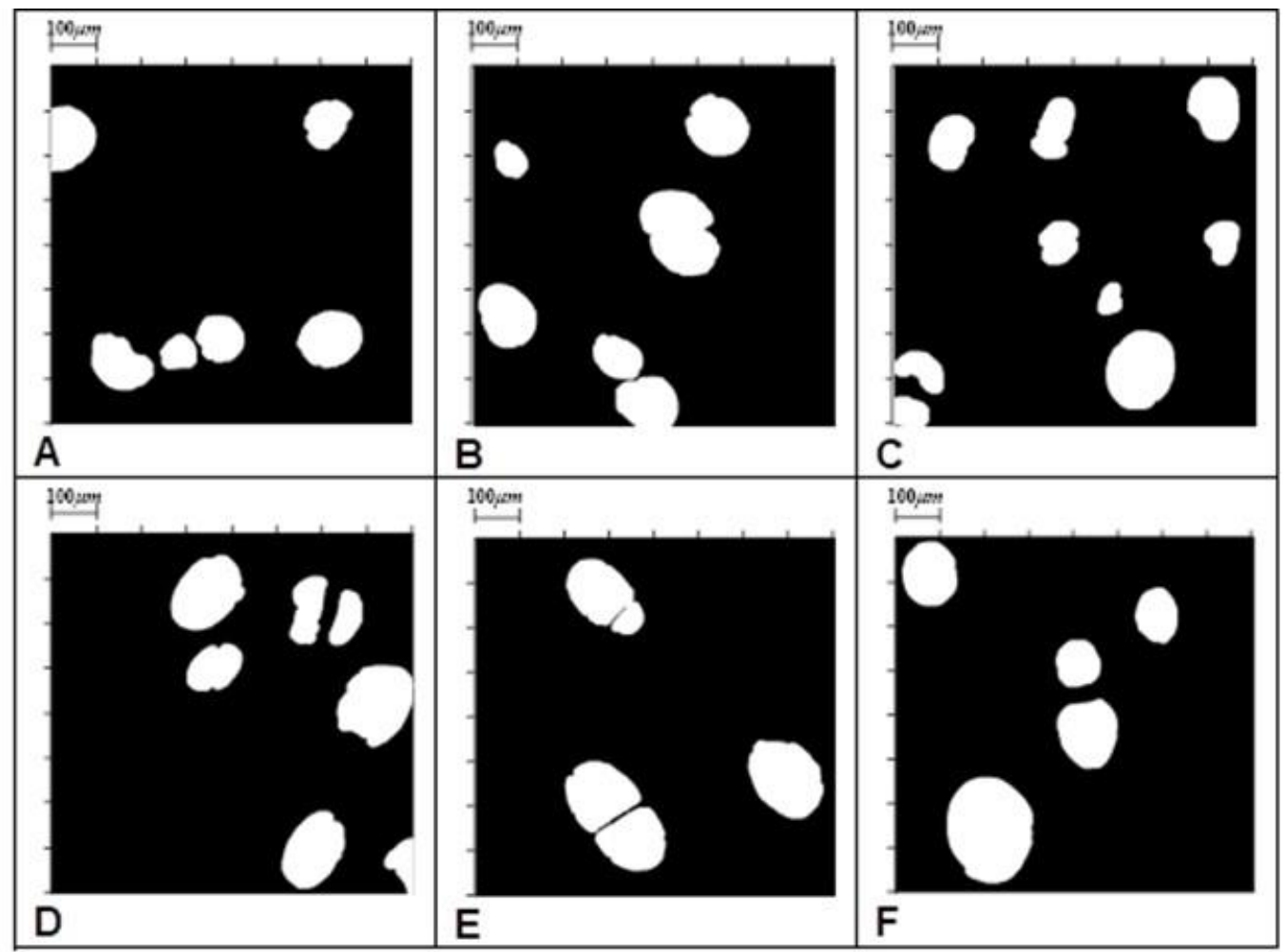

Fig. 2. The binary images after morphological processing: A is Pterocarpus macrocarpus; $B$ is Pterocarpus erinaceus; C is Dalbergia latifolia; D is Dalbergia frutescens; E is Pterocarpus indicus; $\mathrm{F}$ is Pterocarpus soyauxii

Figure 2 shows the operation objects. Using (5)-(11), the following vessel pores parameters were calculated: the number $N$, average area $S_{\text {ave, total area }} S_{\text {sum, average }}$ perimeter $P_{\text {ave }}$, total perimeter $P_{\text {sum, }}$ average roundness $C_{\text {ave }}$, and total roundness $C_{\text {sum }}$. Seven feature parameters were used for classification and recognition by the BP neural network and RBF kernel function support vector machine. The recognition results are shown in Table 2. 
Table 2. Statistical Classification Accuracy after Two Kinds of Feature Extraction

\begin{tabular}{|c|c|c|}
\hline Classification & Accuracy & Running Time \\
\hline BP neural network & $99.4 \%$ & $7 \mathrm{~s}$ \\
\hline RBF-SVM & $98.9 \%$ & $179 \mathrm{~s}$ \\
\hline Note: The best results are bolded & \\
\hline
\end{tabular}

Table 2 shows that the BP neural network for classification is better than SVM, and the accuracy rate is up to $99.4 \%$, and the running time is only $7 \mathrm{~s}$. It also shows that it is feasible to classify and recognize six kinds of hardwood wood species in the Papilionaceae by using the vessel pores' features as input parameters.

In order to better distinguish the wood species with little difference in vessel pores structure, the confusion matrix method was used to analyze the characteristics of six kinds of hardwood species (Class A is Pterocarpus macrocarpus; Class B is Pterocarpus erinaceus; Class C is Dalbergia latifolia; Class D is Dalbergia frutescens; Class E is Pterocarpus indicus; Class $\mathrm{F}$ is Pterocarpus soyauxii). 30 samples of each hardwood species were collected for testing, 180 samples in all. The analysis results are shown in Figs. 3 and 4.

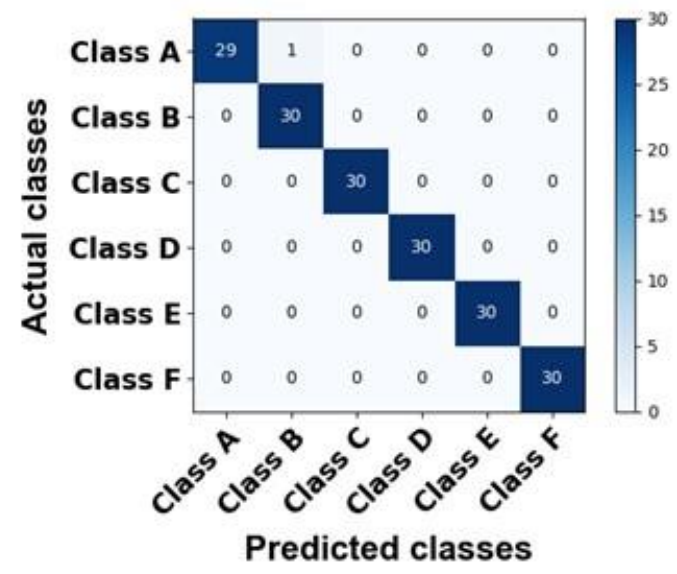

Fig. 3. The confusion matrix after BP neural network classification (Class A is Pterocarpus macrocarpus; Class B is Pterocarpus erinaceus; Class C is Dalbergia latifolia; Class D is Dalbergia frutescens; Class E is Pterocarpus indicus; Class F is Pterocarpus soyauxii. Figures 4 and 6 expression method are the same.

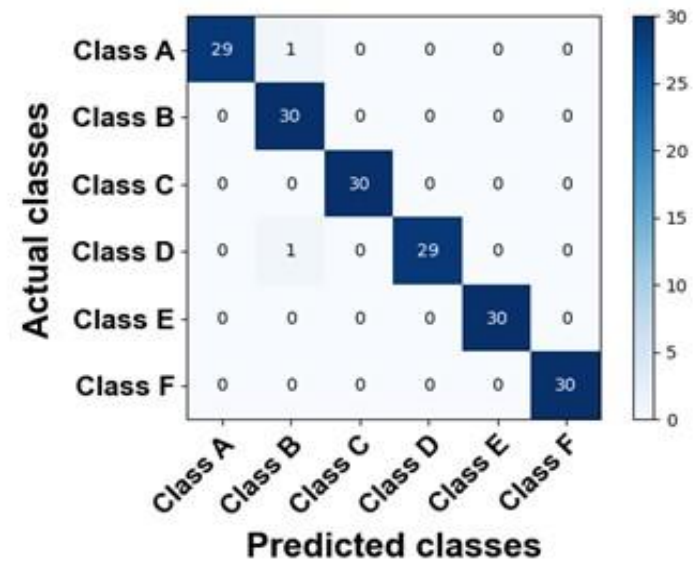

Fig. 4. The confusion matrix after support vector machine classification 
Figure 3 shows the confusion matrix of six kinds of hardwood after classification through BP neural network. There were six categories, and each category had 30 samples. 179 samples were correctly classified, and the accuracy rate was as high as $99.4 \%$. Only one sample in Pterocarpus macrocarpus was mistakenly identified as Pterocarpus erinaceus. Figure 4 shows the confusion matrix of six kinds of hardwood after support vector machine classification. Thirty samples were chosen from each category and 178 samples were correctly classified, and the accuracy rate was as high as $98.9 \%$. One sample in Pterocarpus macrocarpus and one sample in Dalbergia frutescens were mistakenly considered as Pterocarpus erinaceus, respectively. This error was due to the similarity of the number, structure, and size of vessel pores between Pterocarpus macrocarpus and Pterocarpus erinaceus.

In order to analyze the results of confusion matrix comprehensively, to verify the performance of each class from the confusion matrix, TP (True Positive), TN (True Negative), FP (False Positive), and FN (False Negative) were used. True Positive means that the original sample was a positive sample, and the predicted sample was also a positive sample. True Negative indicates that the real sample was negative, and the prediction was also a negative sample. False Positive indicates that the real sample was negative, and the prediction was a positive sample. False Negative means that the real sample was positive, and the prediction was a negative sample. Four indexes (Precision, Recall, $\mathrm{F}_{1}$-Score, and Accuracy) were evaluated. The formulas are shown in (12)-(15). Six kinds of hardwood were classified by the BP neural network and the RBF Kernel SVM classifier, and the evaluation results are shown in Figs. 5 and 6:

$$
\begin{aligned}
& \text { Precision }=\frac{T P}{T P+F P} \\
& \operatorname{Re} \text { call }=\frac{T P}{T P+F N} \\
& F_{1}-\text { Score }=\frac{2 \times \operatorname{Pr} \text { ecision } \times \text { Re call }}{\operatorname{Pr} \text { ecision }+\operatorname{Re} \text { call }} \\
& \text { Accuracy }=\frac{T P+T N}{T P+T N+F P+F N}
\end{aligned}
$$

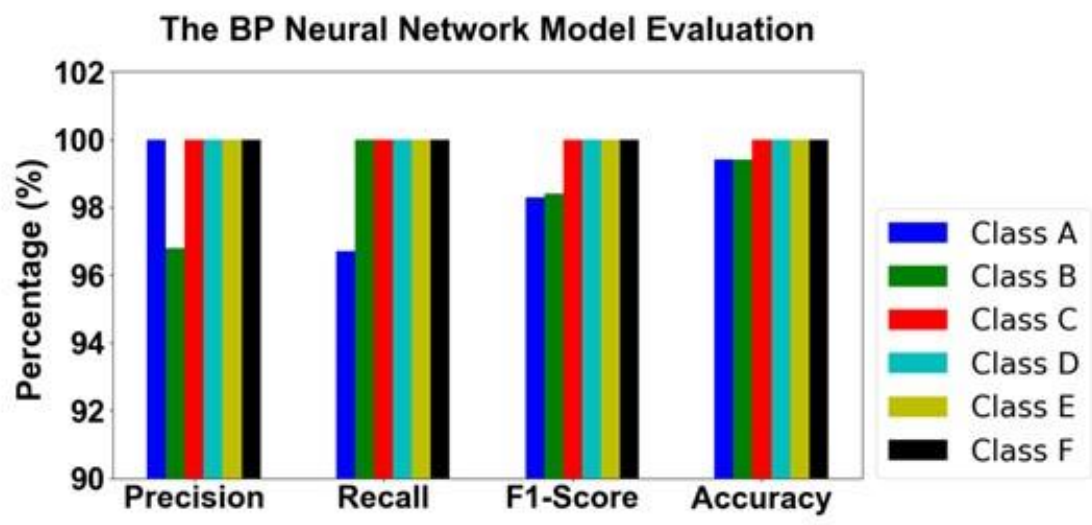

Fig. 5. The model evaluation after the BP neural network classifier 


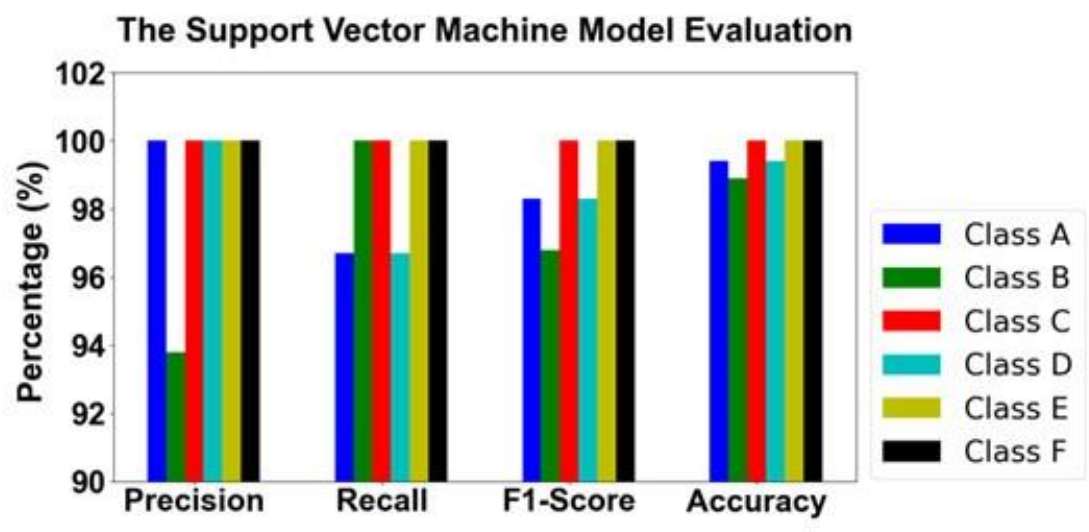

Fig. 6. The model evaluation after the support vector machine classifier

Figure 5 shows the model evaluation chart after BP neural network classification. The recall rate of Pterocarpus macrocarpus was $98.3 \%$, the performance of $\mathrm{F}_{1}$-score was $98.3 \%$, the accuracy rate was $99.4 \%$, the recall rate of Pterocarpus erinaceus was $98.3 \%$, the performance of $\mathrm{F}_{1}$-score was $98.3 \%$, and the accuracy rate was $99.4 \%$. In addition to these evaluation indexes, the other evaluation indexes of the six tree species were $100 \%$.

Figure 6 shows the model evaluation chart after the support vector machine classification. The recall rate of Pterocarpus macrocarpus was $96.7 \%$, the performance of $\mathrm{F}_{1}$-score was $98.3 \%$, and the accuracy rate was $99.4 \%$. The lowest precision of Pterocarpus erinaceus was $93.8 \%$, the performance of $\mathrm{F}_{1}$-score was $96.8 \%$, and the accuracy rate was 98.9\%. The recall rate of Dalbergia frutescens was $96.7 \%$, the performance of $\mathrm{F}_{1}$-score was $98.3 \%$, and the accuracy rate was $99.4 \%$. In addition to these evaluation indexes, the other evaluation indexes of the six tree species were $100 \%$.

The above analysis shows that, in the classification and identification of the vessels, the BP neural network was better than the support vector machine classifier. Although the characteristic parameters of hardwood vessel pores had some influence on the classification results, the four indexes were all more than $93.8 \%$. Combined with the evaluation results of confusion matrix, it is feasible to use vessel pores as the basis of hardwood classification.

\section{CONCLUSIONS}

1. Using micro CT to obtain high-resolution micro images of hardwood, thousands of images can be obtained in a single scanning experiment, and the resolution is as fine as $1.95 \mu \mathrm{m}$. It is a very effective and accurate micro image acquisition method. Combined with the level set geometric active contour model and morphological processing method, the contour extraction of hardwood vessel was realized. Then the vessel parameters were obtained to provide data support for BP neural network and support vector machine to classify wood species.

2. The classification results of BP neural network and support vector machine were evaluated by using confusion matrix and four evaluation indexes (Precision, Recall, F1-Score, and Accuracy), and the classification accuracy rate was above 98.9\%. The results showed that the vessel pores were used as the characteristic parameter to 
complete the classification and identification of six kinds of hardwood. This method is feasible and innovative.

3. In this paper, six kinds of hardwood were used as experimental objects, and good results were obtained by extracting vessel features. In the future, we will gradually increase the number of samples, explore suitable vessel segmentation methods in more hardwoods, and gradually improve the classification and recognition of a variety of similar species.

\section{ACKNOWLEDGEMENTS}

This work was supported by the Natural Science Foundation of Shandong Province, China (Grant No. ZR2020QC174) and the Taishan Scholar Project of Shandong Province, China, Grant No. 2015162.

\section{REFERENCES CITED}

Beeckman, H. (2016). "Wood anatomy and trait-based ecology," IAWA Journal 37(2), 127-151. DOI: 10.1163/22941932-20160127

Brunel, G., Borianne, P., Subsol, G., Jaeger, M., and Caraglio, Y. (2014). “Automatic identification and characterization of radial files in light microscopy images of wood," Annals of Botany 114(4), 829-840. DOI: 10.1093/aob/mcu119

Chen, F. F., and Evans, R. (2010). "Automated measurement of vessel properties in birch and poplar wood," Holzforschung 64(3), 369-374. DOI: 10.1515/hf.2010.057

Chih, C. C., and Lin, C. J. (2011). "LIBSVM: A library for support vector machines," ACM Transactions on Intelligent Systems and Technology 2(3), Article Number 27. DOI: 10.1145/1961189.1961199

Ding, Y. R., Cai, Y. J., Sun, P. D., and Chen, B. (2014). "The use of combined neural networks and genetic algorithms for prediction of river water quality," Journal of Applied Research and Technology 12(3), 493-499. DOI: 10.1016/S16656423(14)71629-3

Ferman, A. M., Tekalp, A. M., and Mehrotra, R. (2002). "Robust color histogram descriptors for video segment retrieval and identification," IEEE Transactions on Image Processing 11(5), 497-508. DOI: 10.1109/tip.2002.1006397

Hu, S. Q., Li, K., and Bao, X. D. (2015). "Wood species recognition based on SIFT keypoint histogram," in: Proceedings of the $8^{\text {th }}$ International Congress on Image and Signal Processing (CISP), Shenyang, China, 702-706. DOI: 10.1109/cisp.2015.7407968

Ibrahim, I., Khairuddin, A. S. M., Arof, H., Yusof, R., and Hanafi, E. (2018). "Statistical feature extraction method for wood species recognition system," European Journal of Wood and Wood Products 76(1), 345-356. DOI: 10.1007/s00107-017-1163-1

Kamal, K., Qayyum, R., Mathavan, S., and Zafar, T. (2017). "Wood defects classification using laws texture energy measures and supervised learning approach," Advanced Engineering Informatics 34, 125-135. DOI: 10.1016/j. aei.2017.09.007 
Li, C. M., Xu, C. Y., Gui, C. F., and Martin, D. F. (2010). "Distance regularized level set evolution and its application to image segmentation," IEEE Transactions on Image Processing 19(12), 3243-3254. DOI: 10.1109/TIP.2010.2069690

Mallik, A., Saavedra, J. T., Fernández, M. F., and Naya, S. (2011). "Classification of wood micrographs by image segmentation," Chemometrics and Intelligent Laboratory Systems 107(2), 351-362. DOI: 10.1016/j. chemolab.2011.05.005

Mu, H. B., Yang, Y., and Ni, H. M. (2016). "Wood defect classification based on support vector machine," International Journal of Control and Automation 11(9), 179-190. DOI: 10.14257/ijca.2016.9.11.16.

Rajagopal, H., Khairuddin, A. S. M., Mokhtar, N., Ahmad, A., and Yusof, R. (2019). "Application of image quality assessment module to motion-blurred wood images for wood species identification system," Wood Science and Technology 53, 967-981. DOI: 10.1007/s00226-019-01110-2

Ruan, X. M., Zhu, Y. Y., Li, J., and Cheng, Y. (2020). "Predicting the citation counts of individual papers via a BP neural network," Journal of Informetrics 14(3), 1-15. DOI: 10.1016/j.joi.2020.101039

Ruffinatto, F., Crivellaro, A., and Wiedenhoeft, A. C. (2015). "Review of macroscopic features for hardwood and a proposal for a new character list," IAWA Journal 36(2), 208-241. DOI: 10.1163/22941932-00000096

Souza, D. V., Santos, J. X., Vieira, H. C., Naide, T. L., Nisgoski, S., and Oliveira, L. E. S. (2020). "An automatic recognition system of Brazilian flora species based on textural features of macroscopic images of wood," Wood Science and Technology 54(4), 1-24. DOI: 10.1007/s00226-020-01196-Z

Wang, S., Wu, T. H., Shao, T., and Peng, Z. X. (2019). "Integrated model of BP neural network and CNN algorithm for automatic wear debris classification," Wear 426427(Part B), 1761-1770. DOI: 10.1016/j. wear.2018.12.087

Wang, H.-J., Qi, H.-N., and Wang, X.-F. (2013). "A new Gabor based approach for wood recognition," Neurocomputing 116, 192-200. DOI: 10.1016/j. neucom.2012.02.045

Xin, J., Zhang, R. J., and Nie, S. D. (2012). "Image segmentation based on level set method," Physics Procedia 33, 840-845. DOI: 10.1016/j. phpro.2012.05.143

Zamri, M. I. P., Cordova, F., Khairuddin, A. S. M., Mokhtar, N., and Yusof, R. (2016). "Tree species classification based on image analysis using Improved-Basic Gray Level Aura Matrix," Computers and Electronics in Agriculture 124, 227-233. DOI: 10.1016/j.compag.2016.04.004

Zhang, C., Xie, Y. C., Liu, D., and Wang, L. (2016). "Fast threshold image segmentation based on 2D fuzzy fisher and random local optimized QPSO," IEEE Transactions on Image Processing 26(3), 1355-1362. DOI: 10.1109/TIP.2016.2621670

Zhao, P., and Wang, C. K. (2019). "Hardwood species classification with hyperspectral microscopic images," Journal of Spectroscopy 2019, Article ID 2039453. DOI: $10.1155 / 2019 / 2039453$

Article submitted: March 19, 2021; Peer review completed: May 9, 2021; Revised version received and accepted: May 26, 2021; Published: June 7, 2021.

DOI: 10.15376/biores.16.3.5329-5340 